\title{
Bousculer pour développer des compétences interculturelles en master MEEF
}

Véronique Lemoine-Bresson

\section{(2) OpenEdition \\ 1 Journals}

Electronic version

URL: http://journals.openedition.org/ripes/3025

DOI: 10.4000/ripes.3025

ISSN: 2076-8427

Publisher

Association internationale de pédagogie universitaire

\section{Electronic reference}

Véronique Lemoine-Bresson, "Bousculer pour développer des compétences interculturelles en master MEEF", Revue internationale de pédagogie de l'enseignement supérieur [Online], 37(1) | 2021, Online since 14 February 2021, connection on 16 February 2021. URL: http://journals.openedition.org/ripes/3025 ; DOI: https://doi.org/10.4000/ripes.3025

This text was automatically generated on 16 February 2021.

Article L.111-1 du Code de la propriété intellectuelle. 


\title{
Bousculer pour développer des compétences interculturelles en master MEEF
}

\author{
Véronique Lemoine-Bresson
}

\section{Introduction}

1 La question de la diversité culturelle des élèves n'est pas nouvelle, mais la façon de la prendre en compte est une problématique éducative actuelle dont l'enjeu relève d'une gageure pour la formation des étudiants futurs enseignants. Les élèves ne peuvent plus être définis à partir de caractéristiques figées et monolithiques, «mais à partir d'une dynamique identitaire qui est elle-même le produit d'actualisations diversifiées des différentes composantes et facettes de l'identité globale » (A. Pretceille, 2017, p. 32). Les étudiants doivent être capables d'opérer un retour réflexif sur leurs propres convictions. Ils doivent pouvoir entrer en contact avec le public hétérogène avec lequel ils travaillent en tant que stagiaires, et accepter que la rencontre puisse leur poser problème. La formation devrait leur permettre de développer des compétences interculturelles (désormais $\mathrm{CI}$ ) pour qu'ils soient en mesure de " prendre en compte la diversité des élèves » (Ministère de l'éducation nationale [MEN], Bulletin Officiel du 25-07-2013).

2 À quoi et comment former les étudiants stagiaires pour qu'ils développent des CI, alors qu'ils produisent des discours souvent angéliques et dénués de dissonances sur les diversités culturelles, qu'ils n'osent pas remettre en question, ou dont ils ne veulent pas se défaire (Dervin et al., 2020, section what to do to take down the walls built by ghosts? Paragr. 4)?

Notre article présente d'une part un dispositif pédagogique universitaire qui veut bousculer pour innover, c'est-à-dire installer des zones d'inconfort pour soi-même et pour les autres (Zembylas, 2010), avec l'objectif de développer des CI. Mis en place en 2018, il compte 74 étudiants de master 2 MEEF d'une université des Hauts-de-France 
inscrits dans un cours de dix heures «Gestions des relations et des interactions entre des cultures différentes ». Ce dispositif aborde la question des microaggressions sociales, raciales et genrées (Sue, 2010) qui peuvent toucher tout un chacun, soit en tant que celui qui subit les effets du discours ou des attitudes d'autrui, ou celui qui les fait subir à autrui.

4 L'article présente d'autre part les résultats d'une étude dans laquelle nous cherchons à savoir comment les étudiants du dispositif développent des CI dans des productions écrites collectives négociées et individuelles quand ils interprètent une microaggression vécue, mise en photographie dans un projet mené à l'université new yorkaise de Fordham (Kyun ${ }^{2}$, 2013). Les productions analysées montrent à quel point les microaggressions sont liées aux contextes. Les résultats font apparaitre que de nouvelles catégories peuvent être constitutives des microaggressions, complémentaires à celles définies uniquement à partir des problématiques dites « raciales » aux USA dans les années 1970, puis élargies à d'autres populations dans les années 1980. Des perspectives de formation concluent l'article.

\section{Le dispositif : cadrage théorique et méthodologique}

\subsection{Spécificités des compétences interculturelles en master 2 MEEF}

5 En master MEEF, les étudiants se retrouvent confrontés à un double obstacle. Le premier résulte du lien entre les $\mathrm{CI}$ et les textes institutionnels. Le référentiel de compétences des enseignants mentionne qu'il faut « prendre en compte la diversité des élèves » (MEN, Bulletin Officiel du 25-07-2013). Il faut également qu'ils accueillent et incluent les élèves désignés par l'institution comme allophones (MEN, circulaire n²012-141 du 2-10-2012). «Respecter autrui » est devenu une priorité parmi les quatre fondamentaux de l'école (MEN, circulaire de rentrée 2019, note de service n²019-087 du 28-05-2019). Ces injonctions leur posent cependant des problèmes de mise en œuvre dans des contextes de pratiques très hétérogènes.

Le second obstacle concerne les représentations que les étudiants ont de la relation entre soi et l'autre. En début de master 2, lorsqu'il est question d'interroger leurs attitudes dans le rapport aux autres, ils se déclarent de façon unanime comme des individus à faibles préjugés vis-à-vis d'autrui (Lemoine-Bresson et al., sous presse). Or, dans les travaux qu'ils produisent ensuite en cours, ils étiquettent spontanément certains élèves de "ceux qui ont des origines", de "Kosovar " par exemple, ne se doutant à aucun moment qu'il peut s'agir là d'une anomalie, tant cette dénomination relève d'un permis à l'assignation d'enfants à qui ils s'arrogent le droit de définir une « appartenance géographique ou une appartenance de groupe » (Bettini, 2017, chap. la construction métaphorique de l'autorité, paragr. 5).

7 Après avoir posé un regard sur des modèles de développement de CI existants, nous décrirons des contenus de formation élaborés pour que les étudiants développent des CI dans un dispositif facilitateur de réflexivité.

8 2.1.1. Des modèles aux approches de développement des compétences interculturelles 
Quand il s'agit d'interroger à la fois la gestion de la diversité culturelle qui, à l'école, sous-entend fréquemment le migrant, l'allophone, ou l'enfant issu de l'immigration, et celle $d u$ respect d'autrui, il est intéressant d'examiner les modèles et les approches existants. Les $\mathrm{CI}$ ont longtemps été un objet privilégié de la didactique des langues, espace où le rapport à l'étranger est exacerbé. L'un des modèles de développement des CI le plus connu est celui de Byram (1997), centré sur la prise de conscience par l'apprenant des aspects importants de la communication en situation interculturelle, comme les savoir-faire, être, agir.

10 À l'inverse de ce modèle qui tient compte des interactions, Spitzberg et Changnon (2009) montrent dans leur revue de littérature que de nombreuses conceptualisations de la notion se centrent essentiellement sur l'individu. Certaines recherches tendent parfois à se transformer en formations avec des activités prêtes à l'emploi (Deardorff, 2020), en vue de construire des CI chez l'apprenant. Quant à Holmes et O'Neill (2010, p. 171), ils interrogent la manière dont l'apprenant peut s'autoévaluer compétent d'un point de vue interculturel. Les auteurs proposent le PEER Model qui aide les étudiants à décrire et comprendre le processus développé. Il articule quatre phases pour interroger une situation de rencontre: Prepare-Engage-Evaluate-Reflect (Holmes et O'Neill, 2010, p. 173).

11 Des recherches de la dernière décennie inscrivent plus largement les CI dans des questions d'éducation et de formation (Bezzari et al., 2019; Dervin, 2016, Dervin et al., 2020; Dervin et Gross, 2016; Ogay, 2001). Elles mettent en avant que les CI font partie des compétences qui ne peuvent pas être automatisées, tant les situations d'interaction présentent des variables inattendues et non réductibles à un modèle fini. On échoue parfois à mobiliser « à bon escient différentes ressources pour répondre à une situation qui est à la fois inédite et complexe » (Rey, 2014, section différents types de compétences, paragr. 8). Ces éléments battent en brèche l'utopie de l'étudiant futur enseignant compétent interculturel.

12 2.1.2. Un paradigme pédagogique à penser: une gageure pour l'enseignant formateur

13 En master MEEF, aider les étudiants à développer des CI relève d'un paradigme pédagogique spécifique qui devrait les amener à s'éloigner des prêts-à-faire illusoires.

D'une part, ce paradigme pédagogique doit permettre aux étudiants d'être outillés pour poser un regard critique et réflexif sur les situations d'interaction qui n'ont pas de format figé. Celles-ci sortent souvent de l'ordinaire et leur formulation ne montre pas toujours une action possible : plutôt questionner que chercher des vérités. D'autre part, ce paradigme doit préparer les étudiants à devenir compétents pour affronter les situations d'interaction, vécues ou à vivre, qui mobilisent ou mobiliseront fréquemment la place des imaginaires, si mouvantes et singulières soient-elles. En effet, selon Bachelard (1938/2011), il peut être nécessaire de développer «une attitude expectante presque aussi prudente vis-à-vis du connu que de l'inconnu, toujours en garde contre les connaissances familières » (p. 14).

15 Pour compléter ces premiers éléments d'un paradigme pédagogique, il est intéressant d'interroger la spécificité des finalités, des contenus et des pratiques du développement de $\mathrm{CI}$ en master MEEF. 


\subsubsection{Développer des compétences interculturelles par un processus réflexif et (auto)critique}

16 Les finalités du dispositif discuté privilégient le contact avec certaines notions théoriques ancrées dans le paradigme de l'interculturalité critique, pensée comme une dynamique où les identités et les pluralités s'actualisent dans les interactions, parfois paisibles, parfois conflictuelles, en contextes situés (Lemoine, 2018; Lemoine-Bresson et al., 2018, p. 27). Ces savoirs sont articulés à des savoir-faire pour interroger les évidences angéliques et analyser ce qui se joue dans les interactions entre les personnes en termes de rapport de pouvoir, de manipulation et d'assignation identitaire.

Par CI, dans le cadre de cet article, il faut donc comprendre qu'il s'agit d'un processus réflexif et (auto)critique qui considère les contextes et la situation de production dans laquelle des interlocuteurs interagissent. Dans cette perspective, «les individus en tant que tels, comptent moins que les rapports qu'ils entretiennent» (A. Pretceille, 2020, p. 30). Les individus qui interagissent, ce sont en premier lieu les étudiants eux-mêmes. Ils trouvent dans les élèves cet autrui qui se présente avec ses propres cadres de référence et les trouble. Mais les étudiants ont parfois du mal à identifier qu'un problème se pose en situation de rencontre entre soi et l'autre et qu'il peut devenir un obstacle à la communication et à l'efficacité des actes (White et Gratton, 2017).

L'objectif est d'amener les étudiants à problématiser la situation, c'est-à-dire identifier le problème par la distanciation du discours commun et de l'expérience non réfléchie. Inspirée des travaux de Fabre (2017), la problématisation a été redéfinie dans notre travail scientifique pour l'approche interculturelle (Lemoine-Bresson et Trémion, 2019). Il s'agit d'un outil d'un point de vue didactique dans le sens où il est question d'amener les étudiants à savoir décrire la situation de rencontre, à sélectionner des éléments pertinents et à émettre des hypothèses sur le rapport entre les personnes afin d'interpréter ce qui se joue dans la rencontre. Les CI demandent de savoir mettre des mots précis sur les phénomènes sociaux pour mieux les décrire, les analyser et les comprendre. En effet, la confrontation directe avec les situations vécues ne suffit pas à développer des $\mathrm{CI}$, dans la mesure où un retour critique sur nos propres discours ou attitudes est rarement fait.

Développer des CI relève d'un processus au long cours qui « vaut le coup car il permet d'approcher le soi et l'altérité [...] et d'aller au-delà des figements » (Dervin, 2016, p. 121). Ce qui interroge le format d'un dispositif pédagogique pour qu'il soit pertinent dans un temps d'enseignement restreint à dix heures.

\subsection{Contenus du dispositif innovant}

20 Le dispositif présenté appartient à la catégorie de projets innovants pour lesquels les pédagogues sont peu sensibles aux modes ou aux injonctions. Ces enseignants font partie de ceux qui «mènent des pratiques innovantes sans jamais les qualifier comme telles» (Cros, 1999, p. 135). Ils sont plutôt convaincus que leurs choix permettent aux étudiants de devenir les acteurs-clés de leur propre formation, profitant des expérimentations innovantes pour mieux apprendre. 


\subsubsection{La fonction des ressources pédagogiques choisies}

21 Le caractère innovant du dispositif est d'abord lié à la fonction des ressources pédagogiques choisies, qui diffère de la fonction d'être des ressources de savoir. La caractéristique première des ressources pédagogiques du dispositif est d'amener les étudiants à sortir de leur zone de confort, à mettre au jour leurs croyances et la vision du monde qu'ils construisent, individuellement et collectivement. Comme le souligne Narcy-Combes (2009, p.95), le travail autour de ces ressources déstabilise les conditionnements des étudiants et favorise la prise de conscience de l'écart entre des représentations initiales et les éléments d'analyse coconstruits. La caractéristique seconde des ressources est de mettre la dimension du discours au centre de l'analyse, car elle donne accès au système symbolique qu'un individu utilise et "révèle sa construction du monde, ses illusions et ses mythes. Le discours indique également les luttes de pouvoir au sein des individus» (Martinez, 2015, p. 62). Il faut cependant reconnaitre qu'il n'est pas simple pour les étudiants de noter cet écart et qu'il est souvent difficile de ne pas se référer à ses propres règles et normes habituelles.

\subsubsection{L'innovation par le risque}

22 En didactique, l'innovation peut prendre la forme de contenus d'enseignement revisités, mais aussi s'attacher au rôle des acteurs didactiques, étudiant et enseignant. Dans notre cas, le dispositif promeut l'articulation des savoirs (convocation de notions théoriques coconstruites) à l'expérience des étudiants mise à distance par la réflexivité. Lison et Justras (2014) soulignent à juste titre que cela est plus facile à dire qu'à faire, dans la mesure où les formateurs « doivent non seulement posséder des connaissances et des compétences disciplinaires [...], mais aussi développer et faire preuve de compétences didactiques et pédagogiques » (p.3). Le dispositif propose l'ouverture d'espaces pédagogiques pour développer des $\mathrm{CI}$ et aller au-delà des idéologies dominantes qui entourent la notion, notamment par des écritures à visée réflexive. Il invite les étudiants à endosser une posture qui les amène à se distancier des prêts-àpenser et des recettes miracles qu'ils aimeraient avoir pour gérer la diversité culturelle des élèves en classe, en vue d'un vivre ensemble idéal.

Ce qui nous amène à inscrire nos manières de former dans un bousculer pour innover. Ce choix rejoint Moloney et Turunen (2020) qui rappellent que les enseignants formateurs "need to encourage their students to take risks, to dig into the hidden, and to recognize and model their own capacity in intercultural compétences" (chap. 9 two teacher educators rethinking practice, paragr. 2).

24 Par innovation, il faut comprendre le choix d'un dispositif qui s'appuie sur la notion de « discomfort » développée par Zembylas en éducation (2010), en tant que moyen qui sert « as a medium for individual and social transformation » (p. 706). Les étudiants, confrontés à des ressources déstabilisantes, ont alors à interroger : «their deeply held assumptions about themselves and others by positioning themselves as witnesses (as opposed to spectators) to social injustices and structurally limiting practices such that they see and act as ambiguous rather than as dualistic (e.g., 'us' and 'them') subjects » (Zembylas, 2010, p. 707).

Ainsi, le dispositif vise à bousculer les étudiants dans leurs représentations afin qu'ils prennent conscience de certains phénomènes sociaux et augmentent leur vigilance dans les interactions avec les élèves, ainsi qu'avec les parents de ceux-ci. 

et étudiante en arts visuels et scéniques à la Fordham University à New York. Il est fortement relayé par les journaux américains et les réseaux sociaux. Kyun donne l'occasion aux étudiants d'effectuer une présentation de soi, appropriée au but de l'interaction dans laquelle ils s'engagent (Amossy, 2015, section la "présentation de soi »: Erving Goffman, paragr. 1), à savoir dénoncer des microaggressions vécues. Les étudiants dénoncent une microaggression vécue sur une pancarte, puis Kyun les photographie. On peut saisir dans quels contextes les microaggressions ont été perpétrées et quelles sont les conditions de leur production, tant elles nous sont familières. On pourrait reprocher au projet de Kim Kyun d'orienter le regard vers une dimension racisante du phénomène des microaggressions. Il faut cependant le replacer dans un contexte où des pratiques et des attitudes ont pour effet « d'actualiser l'idée de race en produisant des individus et des groupes racisés » (Poiret, 2011, p. 113). Seuls les jeux d'opposition Whites versus Afro Americans/Asian Americans et Latino-Hispano Americans sont traités dans le projet de Kim Kyun.

31 Parmi les 20 photos du projet, prenons l'exemple de Michele [Annexe 1]. Les yeux levés au ciel, elle tient une pancarte où est noté : « No, where are you REALLY FROM? ». Quel est l'intérêt pour les étudiants enseignants stagiaires d'interroger le discours de Michele? La question cible de façon directe les origines de la personne. Les marques linguistiques, no en début de phrase, puis really, contribuent à la construction du personnage et d'un interlocuteur; elles laissent supposer qu'une question vient d'être posée à Michele, mais que sa réponse ne satisfait pas le questionneur, qui est souvent un inconnu dans ce genre d'échange. L'indice extraverbal des yeux levés au ciel est également très intéressant. La mauvaise question des origines est aussi la fréquente 
question des origines, et par cette expression, Michele signale sa lassitude à être interrogée sur ce sujet. L'insistance et la fréquence provoquent alors des émotions fortes qui parfois inspirent de la colère. La question n'est pas anodine et les étudiants se sentent bousculés dans leur bienséance à penser quand on leur dit que cela «ne veut rien dire d'autre que de quelle race es-tu? " (Dumitru, 2015, s.p.).

Si on pense qu'il s'agit là d'une provocation et que cela fait sens uniquement aux ÉtatsUnis, il est temps d'écouter et de lire ce que disent certains acteurs de la vie publique en France. L'écrivaine Tania de Montaigne (2018) par exemple, se dit fatiguée de cette question et de ce qu'elle présuppose. Dans l'émission Entrée Libre de France 5 en 2017, l'acteur Jamel Debbouze s'en prend à la journaliste qui lui pose la question qu'il ne fallait pas poser, celle des origines qui, selon Dumitru (2015) «exprime une forme de racisme" (paragr. 24). L'acteur manifeste ouvertement son agacement de cette assignation, qui, selon lui, ne fait aucun sens avec la réalité du monde actuel, et a des effets néfastes sur autrui. Ces témoignages montrent que la question place la personne dans la position de l'étranger perpétuel dans son propre pays.

La question des origines est une problématique cruciale au cœur du métier enseignant, renforcée par les situations actuelles, socialement vives, de l'accueil des enfants migrants qui arrivent dans les écoles. Les étudiants enseignants stagiaires constituent un auditoire intéressant pour analyser ce type de microaggression. Tout d'abord, ils sont déjà en poste à mi-temps, et ensuite, ils se nourrissent de représentations, de normes et de valeurs qu'ils partagent sur l'accueil des élèves. Tous en effet s'accordent à se dire bienveillants et ouverts, sans avoir interrogé leurs façons de faire dans les interactions avec autrui, sans penser que ce qu'ils véhiculent dans leurs discours n'est pas anodin. Ils sont également influencés par les documents institutionnels qui promeuvent une mobilisation autour de l'entente, faisant l'impasse sur les conflits. Comme le souligne Maulini (2019), "vivre ensemble nos diversités exprime un modus vivendi qui peut pousser l'art de s'accorder à son sommet, jusque dans une civilité des conduites dispensant de se disputer, ne serait-ce que par la parole et des marques symboliques de clivage » (p. 2).

Il est fondamental que les étudiants mettent à distance ce que gérer la diversité peut vouloir dire et prennent conscience des effets de la question des origines, tant elle est posée de façon banale et parfois sympathique. Les étudiants justifient cette question par «un désir à la fois de curiosité et d'information» (Lüsebrinck, 1996, p. 54), alors que, selon Dumitru (2015), elle relève d'une "anomalie au regard des pratiques habituelles de la conversation » (s.p.). Ce contraste entre la banalité de la question, liée à l'intention du questionneur, et les effets sur le questionné est un point de vigilance qui peut être soulevé dans le cadre d'un travail universitaire sur le développement des CI.

Une autre photo du projet de Kim Kyun montre Josh [Annexe 1] qui fait la moue. Il se "met en scène dans sa façon de s'habiller et de se comporter, dans ses gestes et ses mimiques" (Amossy, 2015, chap. 4 images de soi, images de l'autre, paragr.1). Il est écrit sur sa pancarte: "The limited representation of my race in your classroom does not make me the voice of all Black people ». Dans ce cas, quel est l'intérêt pour les étudiants enseignants stagiaires d'interroger le discours et l'attitude de Josh? Associée aux mots, sa mimique faite à dessein est une autre information sur laquelle on peut s'appuyer pour interpréter cette microaggression. Josh dénonce la tendance à vouloir faire d'une seule personne la représentante de tout un groupe, uniquement parce qu'elle partage 
un élément commun visible avec ce groupe, ici, la couleur de peau. L'assigné se sent alors devoir être le porte-parole de tout un groupe sous l'imposition des autres. On peut penser, par l'analyse de l'exemple de Josh, que la présentation de soi relève de ce que le sujet veut en faire. Il décide soit de nouer des liens étroits avec un groupe d'appartenance, voire s'en réclame, ou bien à l'inverse, il décide de s'en distinguer. Il est alors crucial que les étudiants stagiaires interrogent les étiquettes collées à autrui en déchiffrant les modalités d'énonciation qu'on ne contrôle pas toujours et qui peuvent avoir des effets sur l'autre.

Selon Kim Kyun, il s'agit d'interpeler la population américaine sur la problématique des microaggressions qui touchent des personnes identifiées aux États-Unis sous les catégories "African Americans ", " Asian Americans » et "Latino/Hispanic Americans » qui sont les plus susceptibles ${ }^{3}$ de subir des microaggressions (Sue, 2010, p. 146-153). Des recherches (Gaertner et Dovido, 1986; Oluo, 2018; Sue, 2010) s'accordent à dire que les microaggressions s'invitent dans tous les espaces sociaux, dont l'école. Elles montrent à quel point les préjugés raciaux sont omniprésents, alors même que l'on se défend d'être racistes, comme le font avec honnêteté les étudiants en début de cours. Pourtant, les croyances racistes façonnent les discours, même et surtout lorsque nous n'en sommes pas conscients et qu'ils ont l'apparence de compliments. Sue (2010, p. 9) explique qu'il est important d'analyser les messages cachés dans les microaggressions pour interroger les stéréotypes et les visions du monde en termes d'inclusion ou d'exclusion, mais aussi de supériorité et d'infériorité dans les rapports sociaux. Son analyse met également au jour les effets sur la santé mentale de la personne assignée, c'est-à-dire victime de microaggressions, fréquentes, invisibles, souvent involontaires. Seule une observation de situations et une analyse des éléments verbaux et extra-verbaux amènent à prendre conscience de ce qui se joue dans l'interaction.

\subsubsection{L'intérêt d'interroger la présentation de soi et la place de l'interlocuteur absent}

Dans le cours "Gestion des relations et des interactions entre des cultures différentes ", les espaces pédagogiques comprennent une sensibilisation des étudiants à l'analyse de la présentation de soi dans les discours des interlocuteurs, qu'ils soient présents ou représentés. Les étudiants sont aussi initiés à la question des mises en scène de soi en situation d'interaction. Cela passe notamment par des échanges et des écritures qui doivent les amener à négocier les interprétations de la situation proposée. Les travaux de Goffman (1973) sont particulièrement éclairants sur la construction de l'image du soi à partir de la «façade personnelle » (p. 30), c'est-à-dire, l'apparence dont le sexe, l'âge, l'habillement, la posture, les expressions du visage, et les manières. Cela donne des indications sur le rôle que l'acteur entend jouer (Amossy, 2015, section la "présentation de soi »: Erving Goffman et l'ordre de l'interaction, paragr. 6). D'une part, il est fait une place importante à ce qui est dit et comment cela est dit, d'autre part à la façon dont les éléments extra-verbaux donnent du sens à la situation. 


\section{Méthodologie de l'étude}

\subsection{Contexte et étudiants} en master MEEF d'une université des Hauts-de-France en 2018. Ces écrits ont été produits en groupes, à la suite du cours sur la notion de microaggression, abordée par un travail sur la vidéo "Microaggressions in everyday life » de Derald Sue (2010) et par la présentation du projet de Kim Kyun décrit plus haut. L'article expose comment les étudiants analysent la présentation de soi, qui peut être une construction d'image volontaire ou involontaire, à partir de l'expression de microaggressions d'étudiants américains qui ont participé au projet de Kim Kyun.

\subsection{Constitution du corpus}

e corpus est double. Il comporte d'une part 22 productions collectives, anonymisées et codées de GR1 à GR22 (18 groupes de 4 personnes et un groupe de 2 personnes). D'autre part, il regroupe 74 productions individuelles, codées avec les initiales de l'étudiant. La consigne de travail est la suivante :

40 - (a) choisissez une photo du projet de Kim Kyun. Justifiez votre choix et analysez le document, c'est-à-dire interrogez ce qui est dit et comment cela est dit.

Dans cette partie, les étudiants écrivent un texte collectif dont ils doivent négocier le contenu.

42 - (b) sur le même mode que celui impulsé dans le projet, écrivez chacun un texte bref qui vous touche directement (photo de vous non obligatoire).

Le contenu peut faire référence à une autre microaggression que la racial microaggression, du moment qu'il permet la réflexion autour de la notion, mais cet élément n'est pas explicitement formulé. La partie (b) de la consigne se veut bousculante dans le sens où elle impose des contraintes dont les étudiants s'emparent (à des degrés variés) pour créer leur propre mise en mots d'une microaggression vécue, au-delà des racial microaggressions imposées dans le projet de Kim Kyun.

Le projet mis en ligne permet de choisir parmi 20 photos, 15 jeunes femmes et 5 jeunes hommes. Seulement environ la moitié des photos est choisie par les 22 groupes d'étudiants. La plus grande concentration de groupes $(4 / 22)$ se fait sur la photo de Winter ("You're really pretty... for a dark skin girl »). Le tableau 1 décrit les photographies dans leur ordre d'apparition sur le site du projet. Il indique les groupes qui ont choisi la photographie référencée, le prénom de l'étudiant de l'université américaine et le texte de la microaggression dénoncée.

Tableau 1. Description des microaggressions du site et choix des groupes

\begin{tabular}{|l|l|l|}
\hline $\begin{array}{l}\text { Groupes } \\
\text { étudiants de } \\
\text { Lille }\end{array}$ & $\begin{array}{l}\text { Prénom de l'étudiant } \\
\text { de l'université } \\
\text { américaine }\end{array}$ & Texte de la microaggression \\
\hline- & Amber & "Why do you sound White?" \\
\hline
\end{tabular}




\begin{tabular}{|c|c|c|}
\hline- & Darby & "You don't act like a normal black person, you know?" \\
\hline GR21, GR22 & Amanda & $\begin{array}{l}\text { "Can you read this?' He showed me a Japanese character on } \\
\text { his phone." }\end{array}$ \\
\hline - & Jaime & $\begin{array}{l}\text { "When I gave a speech about racism, the emcee introduced } \\
\text { me as 'Jaime Garcia.' My name is Jaime Rodriguez; not all } \\
\text { Latinos have the last name Garcia." }\end{array}$ \\
\hline - & Cesca & $\begin{array}{l}\text { "What are you?' Human. Being biracial doesn't make me a } \\
\text { 'what." }\end{array}$ \\
\hline GR19, GR20 & Sandra & "So... you're Chinese... right?" \\
\hline GR18 & Sri & "You don't speak Spanish?" \\
\hline GR16, GR17 & Kristina & $\begin{array}{l}\text { "This girl sitting next to me moves, to sit closer to someone } \\
\text { she's talking to, and this white guy whispers loudly that } \\
\text { she moved because I... "smell like rice" }\end{array}$ \\
\hline GR15 & $\mathrm{Aj}$ & "You're not really Asian" \\
\hline GR14 & Maja & $\begin{array}{l}\text { "Just because I'm Mexican that doesn't mean I should be } \\
\text { the automatic 1st choice for the role of Dora the Explorer } \\
\text { in the high school skit." }\end{array}$ \\
\hline GR12, GR13 & Michele & "No, where are you really from?" \\
\hline - & Aja & $\begin{array}{l}\text { "So what does your hair look like today?' She said as she } \\
\text { pulled off my hat without my permission" }\end{array}$ \\
\hline- & Garrett & "So, like, what are you?" \\
\hline- & Jackie & "Not your fucking China Doll." \\
\hline $\begin{array}{ll}\text { GR9, } & \text { GR10, } \\
\text { GR11 }\end{array}$ & $\mathrm{HP}$ & $\begin{array}{l}\text { "Can you see as much as white people? You know, because } \\
\text { of your EYES..?" }\end{array}$ \\
\hline- & Mika & "So what do you guys speak in Japan? Asian??" \\
\hline GR8 & Courtney & $\begin{array}{l}\text { "Courtney I never see you as a black girl' \#swerve } \\
\text { \#OPENYOUREYES!" }\end{array}$ \\
\hline $\begin{array}{l}\text { GR4, GR5, } \\
\text { GR6, GR7 }\end{array}$ & Winter & "You're really pretty... for a dark skin girl" \\
\hline GR1, GR2, GR3 & Josh & $\begin{array}{l}\text { "The limited representation of my race in your classroom } \\
\text { does not make me the voice of all black people." }\end{array}$ \\
\hline - & Nisarah & $\begin{array}{l}\text { "When people think it's weird that I listen to Carrie } \\
\text { Underwood" }\end{array}$ \\
\hline
\end{tabular}




\subsection{Méthodologie d'analyse} avec leurs expériences, leur métier ou des projections pour leur pratique de classe. Nous examinons également leurs manières de définir les microaggressions et de les recontextualiser. Par ailleurs, nous reconstruisons leur capacité à tenir compte des dimensions dialogiques de la situation présentée sur la photographie :

52 - rôle donné (ou pas) à l'interlocuteur absent de la situation et à son discours potentiel, à partir duquel on peut émettre des hypothèses;

53 - gestion des voix multiples.

54 Ces éléments sont des marqueurs de développement de $\mathrm{CI}$, telles qu'elles ont été spécifiées dans le cadrage théorique. Les résultats construits à partir des discours des étudiants sont mis en regard de la littérature présentée et de la question du développement de $\mathrm{CI}$. Ils montrent que les $\mathrm{CI}$ se pensent en contexte par rapport à une situation complexe à interroger.

\section{Résultats}

\subsection{Le choix de la photographie}

Les 22 textes se répartissent en 3 catégories de justification du choix de la photographie, que nous avons reconstruites :

56 - (1) la banalité quotidienne et intemporelle des situations;

57 - (2) le caractère déstabilisant et/ou choquant de la photographie, le GR17 alliant les deux; 
59 Les catégories (1) et (2) sont représentées de façon égale, avec dix groupes pour chacune. La catégorie (3) rassemble deux groupes pour lesquels la photo déclenche la question du métier et fait émerger des projections pour la classe (GR3 et GR14). Trois groupes s'appuient sur la photo choisie pour amener des pistes pour l'école (GR4, GR5, GR11). Les éléments qui constituent les catégories ne sont cependant pas uniformes, bien au contraire.

\subsubsection{La banalité quotidienne de situations intemporelles}

60 Même si le projet de Kim Kyun met en avant des pratiques sociales étasuniennes, la plupart des groupes qui parlent de banalité quotidienne de la situation ajoutent : «en France ou partout dans le monde ». S'ils soulignent que c'est une véritable question de société, le groupe 5 dit que cela "a toujours été d'actualité », et " que ce problème est toujours présent au $21^{\mathrm{e}}$ siècle ", s'appuyant sur des événements historiques distants temporellement autour des personnalités de Luther King et Obama. Cette remarque du groupe 5 amène à dire que le projet de Kim Kyun ne révèle pas l'existence du phénomène, mais le (re)met au-devant de la scène en tant que problème vieux comme le monde. Certains indiquent que les microaggressions dénoncées relèvent du racisme ordinaire également en pratique en France, comme le montrent les nombreuses plateformes avec des témoignages. Pour ces groupes, la notion de microaggressions partage avec le racisme ordinaire le fait que l'interlocuteur n'a pas forcément l'intention d'être haineux. Certains groupes incluent dans cette banalité leur propre expérience, le plus souvent dans la position de celui qui a subi une microaggression. Certains s'auto-désignent ou s'inter-désignent comme " ayant des origines étrangères " (GR22), sans relever cependant la dimension essentialisante de leur appréciation, alors qu'ils la dénoncent dans la microaggression vécue par Amanda.

\subsubsection{La zone de confort parfois touchée}

61 Le terme « choquant » est utilisé par quatre groupes sur dix. Par exemple :

62 1. Nous avons choisi cette photo, car c'est celle qui a suscité la plus forte réaction dans notre groupe. On peut même la qualifier de choquante (photo de Kristina, GR16)

Les autres groupes avancent les mots «brutalité », «bouleversant», «fort» ou " contrasté » pour désigner l'attitude de l'étudiante sur la photographie (Michele et Winter). Les étudiants expliquent le choix de ces deux photos en s'appuyant sur des indices multiples qui relient des éléments de la façade personnelle et la place d'un interlocuteur potentiel. Les trois premiers mots "brutalité », «bouleversant " et «fort» sont associés à la déclaration d'un choc négatif, souvent mis en lien avec du vécu. Le quatrième mot « contrasté » est utilisé pour parler de la photo de Winter ou de Michele qui interpelle les groupes 8 et 12, parce que l'attitude enjouée de Winter ou à l'inverse très rebutée de Michele contraste avec les autres photos et fait naitre des questionnements. Par exemple, pour le GR12 :

2. Nous avons choisi cette photo car la femme présente semble avoir un air excédé (elle lève les yeux au ciel), ça nous a particulièrement interpelés. Nous pouvons supposer que les personnes qui discutent avec elle lui demandent très souvent d'où elle vient [les étudiants poursuivent par un scénario d'interactions imaginaires entre Michele et des interlocuteurs potentiels] (GR12) 
65 La photo en tant que ressource vient troubler chez les étudiants ce que Zembylas et Papamickael (2010) appellent "their emotional comfort zones » (p. 3) allant jusqu'à créer de l'empathie, selon les déclarations des groupes 2 et 20. Le trouble les amène à questionner leurs croyances, leurs habitudes et leurs pratiques sociales qui véhiculent des stéréotypes, pour ensuite ouvrir des portes vers la reconsidération de leurs propres convictions.

\subsubsection{Le lien avec le métier d'enseignant}

66 Seuls deux groupes font un lien explicite entre la photographie choisie et le professionnel. Les photos choisies (Josh et Maja) induisent en effet la possibilité de faire ce lien puisqu'elles évoquent des microaggressions en classe. Trois autres groupes indiquent que la photo choisie leur donne des idées pour des pistes professionnelles, que ce soit au niveau micro (la classe), méso (l'établissement) ou macro (l'école). Certains endossent le rôle de l'acteur principal dans la sensibilisation des microaggressions auprès des élèves, d'autres précisent le contexte disciplinaire (« en éducation morale et civique ", GR3, GR11) ou l'insèrent dans un contexte plus large, posant de manière évidente et déclarative la garantie du respect d'autrui assurée par l'école. Selon, les groupes 4 et 5 :

67 1. En tant qu'enseignants, c'est à nous d'apprendre aux enfants à être respectueux et tolérants avec les autres, et ce, dès le plus jeune âge (GR4)

68 2. À travers l'éducation morale et civique, l'éducation a son rôle à jouer. L'école veille à ce que chaque individu soit respecté (GR5)

\subsection{Vision d'ensemble des compétences interculturelles développées ou non}

69 Une partie de notre analyse a cherché à voir si les étudiants, pour interpréter la photographie choisie, ont :

70 (a) pris en compte la dimension du discours (repérages effectués dans le texte de l'étudiant américain) et les mises en scène de soi, notamment ce que Goffman (1973) appelle « la façade personnelle » (p.30) de l'étudiant du projet de Kim Kyun, c'est-àdire, par exemple :

71 - le vêtement : « il (Josh) affiche un côté plus fun avec son bonnet original » (GR1)

72 - l'âge : «il (Josh) a un visage adolescent et donne l'impression d'être beaucoup plus jeune que les autres étudiants, ce qui donne encore plus de force à son propos » (GR2)

73 - les caractéristiques raciales ${ }^{4}$ : « le terme les Blancs est utilisé pour montrer qu'elle (HP) est différente de par sa couleur de peau » (GR9)

74 - l'attitude : «nous observons que la personne (Michele) sur la photo a la tête de côté » (GR3)

75 - les mimiques : « elle (Michele) lève les yeux aux ciels et ne sourit pas » (GR13)

76 - les gestes (ou le gestuel): «nous voyons sur cette photo une jeune fille (Sandra) pointant du doigt le message » (GR19). 
77 (b) pris en compte la voix de l'interlocuteur absent, par exemple quand le groupe 11 dit, à partir de la photographie de HP, « on ressent une certaine innocence de la part de la personne qui dit cette phrase ».

Le tableau 2 synthétise pour chacun des groupes la présence ou l'absence des points (a) et (b) dans les interprétations.

Tableau 2. Prise en charge des dimensions de la façade personnelle et de la place de l'interlocuteur absent

\begin{tabular}{|l|l|l|l|l|l|l|l|l|l|l|l|l|l|l|l|l|l|l|l|l|l|l|}
\hline $\mathrm{GR}$ & 1 & 2 & 3 & 4 & 5 & 6 & 7 & 8 & 9 & 10 & 11 & 12 & 13 & 14 & 15 & 16 & 17 & 18 & 19 & 20 & 21 & 22 \\
\hline (a) & $\mathrm{x}$ & $\mathrm{x}$ & $\mathrm{x}$ & - & $\mathrm{x}$ & $\mathrm{x}$ & $\mathrm{x}$ & $\mathrm{x}$ & $\mathrm{x}$ & $\mathrm{x}$ & - & $\mathrm{x}$ & $\mathrm{x}$ & - & $\mathrm{x}$ & - & - & $\mathrm{x}$ & $\mathrm{x}$ & $\mathrm{x}$ & $\mathrm{x}$ & - \\
\hline (b) & - & - & - & $\mathrm{x}$ & $\mathrm{x}$ & - & - & $\mathrm{x}$ & - & $\mathrm{x}$ & $\mathrm{x}$ & $\mathrm{x}$ & $\mathrm{x}$ & $\mathrm{x}$ & $\mathrm{x}$ & $\mathrm{x}$ & $\mathrm{x}$ & - & $\mathrm{x}$ & $\mathrm{x}$ & - & $\mathrm{x}$ \\
\hline
\end{tabular}

On remarque que tous les groupes prennent en charge au moins un des deux points et que 8 groupes sur les 22 associent les deux (parties grisées).

Neuf groupes sur 22 interrogent les problématiques de l'altérisation ou encore «Otherization» (Holliday et al., 2004) en prenant appui sur l'un ou l'autre des points a) ou b), ou sur les deux en alternance. Ces groupes examinent le processus de mise à distance entre "Us» vs "Them », qui pose une frontière symbolique et des normes ("plus jolie vs moins jolie», dans l'analyse de la photo de Winter, par exemple). « Nous » pouvant être « les Blancs » et « eux » « les Noirs », selon les termes utilisés par certains groupes d'étudiants, critiques de ces désignations qui selon eux marquent la relation dominant-dominé, supérieur-inférieur. Ces analyses peuvent être éclairées d'une part par les travaux de Sue (2010) qui montrent que «microaggressions reflect a biased worldview of superiority-inferiority, and inclusion-exclusion in favor of Whites, and unfavorable attitudes and beliefs toward people of color»(p.157). D'autre part, les commentaires des étudiants font écho aux questions actuelles posées par l'écrivaine Tania de Montaigne (2018) qui veut faire réfléchir au phénomène d'essentialisation de la personne à travers l'utilisation du $\mathrm{N}$ majuscule dans l'expression les Noirs.

81 Dans les 13 groupes restant, on trouve la plus classique trilogie stéréotypes-préjugésdiscrimination que Leyens (2012) désigne comme un des processus « étant les supports du racisme » (p. 70). Certains groupes analysent la relation entre les trois termes (GR16, GR13, GR14), mais la plupart se contentent de parler de l'un ou de l'autre, sans les articuler ni les définir de façon suffisamment précise.

\subsection{Le cas du groupe 20}

Pour cet article qui interroge le développement de $\mathrm{CI}$, nous présentons plus en détail l'analyse de la production du GR20 qui travaille à partir de la photographie de Sandra : «So..., you're chinese, right?» [Annexe 1]. Cette production fait partie des textes qui prennent fortement en compte les dimensions de la façade personnelle en les articulant à la place de l'interlocuteur absent et à des éléments théoriques constitutifs de la définition de la notion de microaggressions. Ce groupe de quatre étudiantes montre également les effets de la photo sur leurs propres convictions et leurs attitudes, et émet des hypothèses sur leur existence. Tous les groupes ont fait la partie b) de la consigne 
(cf. 3.2). Mais le GR20 est l'un des groupes qui a fait la partie (b) en joignant une photo personnelle à la phrase d'amorce, ainsi qu'un texte d'environ 15 lignes, pour chacune des quatre étudiantes.

Il articule d'emblée des éléments de la façade personnelle avec des repères linguistiques et le rôle d'un interlocuteur potentiel pour construire le sens de la situation dénoncée par Sandra. Le texte fait preuve de polyphonie dans la mesure où les étudiantes convoquent dans leur discours la voix des médias, celle de l'éducation à la maison et à l'école et des références scientifiques pour analyser la microaggression. Cette mise au jour des diverses voix leur fait prendre conscience, selon leur déclaration, qu'elles-mêmes auraient pu prononcer cette phrase. Elles se posent en tant qu'assignatrices potentielles. Elles font alors référence à leur expérience au restaurant asiatique où elles disent que "si les personnes ont un faciès asiatique, c'est ce qui garantit pour nous l'authenticité de la cuisine servie ». Quand elles analysent la photo, elles imaginent l'interlocuteur absent en le mettant immédiatement en scène. Elles indiquent que l'interlocuteur absent se sert de repères visuels pour étiqueter Sandra, et que cet interlocuteur attend que Sandra réponde à ce qu'il imagine qu'elle doit être, à savoir, selon les quatre étudiantes, "celle qui a un accent identique à tous les asiatiques », « celle qui mange du riz avec des baguettes ». Le groupe interroge ce qu'A. Pretceille (2020) appelle «la validité des définitions catégorielles comme l'ethnie, la culture, le groupe social » (p. 28), au lieu de replacer la signification des choses au cœur des interactions. Il analyse l'influence de la conception de la culture de l'interlocuteur imaginé sur sa manière de voir l'autre et souligne que cette conception relève du culturalisme dans la mesure où il établit systématiquement un lien entre les attributs physiques visibles et une identité.

84 Au-delà de cette dénonciation, le groupe se penche sur les effets de la microaggression subie par Sandra en avançant : « Mettons-nous à la place de cette personne qui ne passe pas une journée sans entendre ce genre de réflexion, même dite avec bonne foi ». Le groupe parvient au final à circonscrire la notion de microaggressions à partir de caractéristiques comme la fréquence, le fait que le phénomène touche tout le monde, la place et le rôle de l'assigné et/ou de l'assignateur, la non conscience de faire du mal et en même temps la réalité de faire du mal, le fait de poser une question dérangeante.

En conclusion de leur écrit, les étudiantes dénoncent les microaggressions qui réduisent l'identité de Sandra à « une somme de caractéristiques sui generis » (A. Pretceille, 2020, p. 29), en indiquant avec l'appui d'un article de Dervin étudié en cours, que «c'est la relation entre deux personnes elles-mêmes complexes qui va créer une situation unique ». La présence de ce dernier point peut être interprétée comme donnant des indications sur le choix de l'image par les étudiantes, l'image relevant d'un choix délibéré pour pouvoir faire preuve de leur propre développement en terme de $\mathrm{CI}$. Ce qui tend à interroger la pérennité des $\mathrm{CI}$ développées dans le contexte institutionnel, et la mobilisation de ces compétences dans un autre contexte et une situation de production différente.

\subsection{La production individuelle}

Cette partie brièvement traitée se focalise sur un résultat intéressant pour la profession enseignante: en effet les analyses des productions individuelles font émerger le manque d'aura de la profession dans le grand public, selon les déclarations des 
étudiants. Sur les 74 productions individuelles (trois en annexe 2), 30 sont accompagnées d'une photo de l'étudiant (élément facultatif, cf. consigne en 3.2.), 39 sont sans photo et 5 présentent une image en lien avec le texte proposé. Un étudiant présente sa production en anglais. Parmi les étudiants qui n'ont pas joint de photo d'eux-mêmes, certains justifient ce choix en expliquant que la microaggression qu'ils ont choisie de dénoncer ne les concerne pas directement, mais plutôt une connaissance.

L'analyse des 74 productions met au jour 3 catégories dominantes pour 47 textes individuels, et des éléments disparates pour les 27 autres (questions d'obésité, d'âge, de physique ou d'activité sportive). Deux des 3 catégories dominantes convergent avec les thèses de Sue (2010), à savoir les "racial microaggressions", avec une nuance régionaliste, pour 12 textes, et les "gender microaggressions" pour 20 écrits. Des productions entrent parfois dans deux catégories. Pour exemples, quelques phrasesclés :

1. J'aime pas les arabes mais toi je t'aime bien (étudiant PL)

2. T'es Ch'ti t'es forcément mariée avec ton cousin, non? (étudiante M-AW)

3. T'es haute comme trois pommes, c'est mignon (étudiante SD).

Sue (2010), qui rappelle que Chester Pierce dans les années 1970 a conceptualisé le concept de "racial microaggressions", souligne que de nos jours: "it is clear that microaggressions can be expressed toward any marginalized group in our society; they can be gender-based, sexual orientation-based, class-based, or disability-based » (p. 5) Une catégorie non présente dans les travaux américains émerge. On pourrait l'identifier sous la dénomination de profession-orientation microaggressions. En effet, même si Sue (2010) parle de microaggressions dans le milieu du travail, sous le terme de «microinequities» (p.24), la question du genre ou de l'African American/Asian American ou Latino-Hispano American y est toujours associée. Quinze productions dénoncent les microaggressions faites aux enseignants, sans autre attribut, menées par la société, mais aussi par la famille ou les amis. Pour exemples, quelques phrases-clés :

1. C'est bien que tu sois prof, en tant que femme, ça te laisse du temps pour ta vie de famille (étudiante LC)

2. Tu es professeur des écoles? Mais, tu es toujours en vacances, non? (étudiante JT)

Ces déclarations semblent faire écho au discours commun véhiculé par les médias qui affichent que les enseignants français sont les plus mal aimés du monde. Peu de recherches, en France ou ailleurs étudient actuellement cette microaggression spécifique qui émerge des écrits étudiants faits dans un contexte de production unique. Mais la France ne semble pas avoir le monopole de cette question. Des travaux suisses montrent que «le traitement médiatique de la profession d'enseignant est fortement chargé d'émotions. Cela ne facilite pas la compréhension du quotidien des pédagogues » (Boller et Plüss, 2010, p. 30). Ainsi il apparait que le travail sur le projet de Kim Kyun a permis aux étudiants de mettre des mots sur un malaise persistant et d'interroger la manière dont leur identité professionnelle est essentialisée par autrui et parfois réduite à quelques attributs dévalorisants. 


\section{Discussion conclusive}

95 Dans leur dernier article, Dervin et al. (2020) proposent que la formation des futurs enseignants s'appuie sur le principe "Questioning the unquestionables». Dans notre étude, ce qui semble incontestable aux étudiants relève d'une position qui tend à nier qu'ils pourraient avoir envers certains élèves des actes peu louables, parfois identifiables sous le terme de racisme ou même de "sexisme bienveillant " (Leyens, 2012, p. 125), quand on demande aux garçons de la classe de prendre en charge une tâche qu'on estime non adaptée aux filles, par exemple. C'est incontestable, ils vont devenir des compétents interculturels pour gérer la diversité des élèves, comme l'indique le référentiel de compétences de la profession. Cet incontestable est rarement questionné, et encore moins dans un versant déstabilisant et bouleversant. Alors que les actes déhumanisants comme les microaggressions font le quotidien de nombreuses personnes, nous sommes inconscients de leurs effets sur autrui. Il est cependant crucial pour les étudiants de développer des CI qu'ils pourraient mobiliser afin d'analyser des situations en contexte éducatif et de mettre au jour ce qui parfois " prive autrui d'une partie de son humanité " (Leyens, 2012, p. 152). Des expressions comme celles des étudiants du projet de Kim Kyun circulent dans la société en toute bonhommie. Le dispositif présenté veut bousculer les prêts-à-penser des étudiants stagiaires pour qu'ils s'impliquent dans ces discours qui circulent dans les situations les plus anodines, notamment à l'école.

Le choix du projet photographique n'est cependant pas neutre, dans la mesure où il se passe aux États-Unis. L'idée est de proposer un travail à partir d'une autre façon de voir le monde. En effet, les jeux d'opposition Whites versus Afro Americans/Asian Americans/ Latino-Hispano Americans prévalent aux États-Unis. Ils font sens dans un « rapport social de domination-subordination qui fonctionne par référence aux origines » (Poiret, 2011, p. 108). Tout comme l'utilisation du mot Race déstabilise : il n'existe pas de races, mais il en existe bien un usage social. Les étudiants de l'étude, à l'exception des groupes 5 et 15 , font peu de cas de ces aspects du contexte, qui donnent tout son poids à la situation de production. Le GR5 replace son usage social dans l'histoire des États-Unis, tandis que le GR15 propose une analyse politique et sociologique. On sait bien qu'en France (ou encore en Europe), les jeux d'opposition concernent plutôt les immigrés, les migrants, les Roms ou comme le dit le groupe 3, « les arabes, les blondes, les Belges, les riches, les pauvres ». Le GR3, sans aller loin dans le questionnement, indique qu'il est également urgent de remettre en cause la domination qui traverse les oppositions autres que Whites/Blacks, comme celle riches/pauvres (Michaels, 2006).

Par ailleurs, afin de ne pas enfermer les étudiants dans un travail à partir des racial microaggressions (les seules présentes dans les 20 photos du projet), nous avons opté, en proposant la partie (b) de la consigne, pour ce que Dervin et al. (2020) donnent comme second principe d'une formation au développement de CI en éducation, à savoir "The mirror: turning inward ». Chaque étudiant s'est saisi de cette opportunité pour interroger une microaggression vécue. La grande majorité s'est désignée comme assignée $(n=70)$. Rappelons cependant que la consigne demandait de s'inspirer du travail fait dans le projet de Kim Kyun. Un étudiant dit ne pas avoir vécu de microaggression, et trois se sont posés comme les assignateurs ("nous pouvons être les instigateurs», GR13). Enfin, de ce corpus, émerge une nouveauté qui s'écarte de la dimension racisante du projet de Kim Kyun, et met au rang des préoccupations les ressentis des étudiants (15/74) sur la 
considération sociale de leur métier. Ce résultat ouvre des perspectives de recherche et d'actions, pour donner la parole à des acteurs peut-être pas suffisamment écoutés.

Il n'est donc pas vain de mettre en place un dispositif qui bouscule les futurs enseignants en les poussant dans leur zone d'inconfort, mais aussi qui pose des contraintes les amenant à créer une production originale. Cela permet à tous les étudiants de se frotter aux réalités et de s'exercer à développer des CI qui leur permettent de rester vigilants quant aux phénomènes hiérarchisants de valorisation/ dévalorisation d'autrui qui peuvent se déguiser sous forme de compliment ou d'humour. Enfin, le choix de recourir à un projet photographique pour développer des $\mathrm{CI}$ incite les étudiants à prendre des repères dans les éléments visuels, discursifs ou extratextuels et à recourir à des voix absentes, et ainsi à interroger sous divers angles une situation complexe qui n'a d'intérêt qu'en contexte, dans une situation de production précise.

En prolongement, on pourrait continuer, dans un temps de formation idéal, à développer des $\mathrm{CI}$ par la confrontation des diverses productions à partir d'une même photographie. Les étudiants pourraient prendre conscience de la pluralité des interprétations. Ils pourraient comprendre qu'interpréter une situation complexe ne va pas de soi et que toute interprétation peut être relativisée, mais non qualifiée de défaillante. Ce qui n'empêche pas de partager des savoirs communs sur les problématiques de société, comme les microaggressions, et d'en faire un objet d'attention et de déconstruction. Pour conclure, l'étudiant futur enseignant en tant qu'individu en voie de devenir compétent interculturellement «ne peut pas être conçu comme un système d'interactions mécaniques avec son environnement. Il doit être vu comme capable d'actions intentionnelles" (Rey, 2014, section pour conclure dans: C. Une conception du sujet, paragr. 2) pour prendre conscience de ses points d'amélioration dans le rapport à autrui.

\section{BIBLIOGRAPHY}

A. Pretceille, M. (2020). La communication interculturelle. Entre pertinence et impertinence. L'Harmattan.

A. Pretceille, M. (2017). Quelle école pour quelle intégration? Hachette.

Amossy, R. (2015). La présentation de soi. PUF.

Bachelard, G. (1938/2011). La formation de l'esprit scientifique. VRIN.

Bettini, M. (2017). Contre les racines (traduit par P. Vesperini). Champs Actuels.

Bezzari, S., Sanojca, E. et Eneau, J. (2019). Repérer les compétences collaboratives et les compétences interculturelles en formation d'adultes. Éducation permanente, 1(218), 143-160.

Boller, B. et Plüss, S. (2010). Dans la presse suisse. Éducateur, 3, 27-30.

Byram, M. (1997). Teaching and assessing intercultural competence. Multilingual Matters. 
Cros, F. (1999). Autour des mots. L'innovation en éducation et en formation dans tous les sens. Recherche et Formation, 31, 127-136.

Deardorff, D. K. (2020). Manuel de développement des compétences interculturelles : les cercles d'histoires. UNESCO. https://unesdoc.unesco.org/ark:/48223/pf0000372192/PDF/

372192fre.pdf.multi

Dervin, F. (2016). Compétences interculturelles. Éditions des Archives Contemporaines.

Dervin, F., Cheng, A., Yuan M. et Jakobsson, A. (2020). COVID-19 and interculturality: first lessons for teacher educators. James Nicholas Publishers. DOI: https://doi.org/10.7459/es/38.1.06

Dervin, F. et Gross, Z. (2016). Intercultural competence in education: Alternative approaches for different times. Palgrave Macmillan.

Dervin, F., Moloney, R. et Simpson, A. (2020). Intercultural competence in the work of teachers. Confronting ideologies and practices. Routledge.

Dumitru, S. (2015). De quelle origine êtes-vous? Banalisation du nationalisme méthodologique. Terrains/Théories, 3, 1-15.

Fabre, M. (2017). Qu'est-ce que problématiser? VRIN.

Gaertner, S.L. et Dovido, J.F. (1986). The adversive form of racism. Dans J-F. Dovido et S-L. Gaertner (dir.), Prejudice, discrimination and racism (p. 61-89). Academic Press.

Goffman, E. (1973). La mise en scène de la vie quotidienne. 1. La présentation de soi. Les Éditions de Minuit.

Holliday, A., Hyde, M. et Kullman, J. (2004). Intercultural communication. Routledge.

Holmes, P. et O’Neill, G. (2010). Autoethnography and self-reflection: tools for self-assessing intercultural competence. Dans Y. Tsai et S. Houghton (dir.), Becoming intercultural. Inside and outside the classroom (p. 167-191). Cambridge Scholars.

Kyun, K. (2013). Racial microaggressions. https://nortonism.tumblr.com/

Lemoine V. (2018). L'interculturel en réflexion pour la classe et ailleurs. RED, 25, 77-92.

Lemoine-Bresson, V. et Trémion, V. (2019, 3-5 juillet). Analyse filmique dans la formation à l'interculturalité des futurs enseignants. Congrès International de l'AREF. Université de Bordeaux, Bordeaux, France.

Lemoine-Bresson V., Lerat S. et Gremmo M.J. (2018). (Dé)construction de la notion d'interculturalité par les étudiants, futurs enseignants. RED, 26, 25-40.

Lemoine-Bresson V., Lerat S., Trémion, V. et Gremmo M.J. (sous presse). Quelles représentations de l'interculturalité chez des étudiants futurs enseignants? Dans V. Delorme et A. Bretegnier (dir.), L'interculturel dans l'enseignement supérieur : enjeux, conceptions, pratiques et dispositifs. Édition Archives Contemporaines.

Leyens, J. P. (2012). Sommes-nous tous racistes? Psychologie des racismes ordinaires. Mardaga.

Lison, C. et Justras, F. (2014). Innover à l'université : penser les situations d'enseignement pour soutenir l'apprentissage. RIPES, 30(1), 1-8.

Lüsebrick, H. J. (1996). La perception de l'Autre. Tangence, 51, 51-66.

Maulini, O. (2019). Que penser... de la diversité à l'école? [Série « Que penser...? »]. Université de Genève, Faculté de psychologie et des sciences de l'éducation, Genève, Suisse.. https:// www.unige.ch/fapse/SSE/teachers/maulini/publ-1906.pdf 
Martinez, V. (2015). Construction de l'est et de l'ouest : vers des compétences interculturelles? [thèse de doctorat, Université de Turku, Finlande]. Annales universitatis Turkuensis. https:// www.utupub.fi/bitstream/handle/10024/118573/AnnalesB413Martinez.pdf? sequence $=2 \&$ is Allowed $=y$

Michaels, W. B. (2006). La diversité contre l'égalité. Raisons d'Agir Éditions.

Ministère de l'éducation nationale. (2019, 28 mai). Circulaire de rentrée 2019, note de service n²019-087 du 28-05-2019 : les priorités pour l'école primaire. Gouvernement de France. https:// www.education.gouv.fr/bo/19/Hebdo22/MENE1915810c.htm

Ministère de l'éducation nationale. (2012, 02 octobre). Circulaire n²012-141 du 2-10-2012 : Organisation de la scolarité des élèves allophones nouvellement arrivés. Gouvernement de France. https://www.education.gouv.fr/bo/12/Hebdo37/MENE1234231C.htm

Moloney, R. et Turunen, T. (2020). Two teachers educators re-thinking practice: intercultural competences in teaching education pedagogy. Dans F. Dervin, R. Moloney, et A. Simpson, (dir.), Intercultural competence in the work of teachers. Confronting ideologies and practices (Part. III, chap. 9, version Kindle). Routledge.

Montaigne (de), T. (2018). L'assignation. Les Noirs n'existent pas. Grasset.

Narcy-Combes, F. (2009). Développer la compétence interculturelle : un défi identitaire. Recherche et pratiques pédagogiques en langues de spécialité, xxviii(1), 93-104. https://doi.org/10.4000/apliut. 1239

Ogay, T. (2001). De la compétence à la dynamique interculturelle. Peter Lang.

Oluo, I. (2018). So you want to talk about race. Searl Press.

Poiret, C. (2011). Les processus d'ethnicisation et de raci(ali)sation dans la France contemporaine : Africains, Ultramarins et « Noirs ». Revue européenne des migrations internationales, 27(1), 107-127.

Rey, B. (2014). La notion de compétence en éducation et en formation. Enjeux et problèmes. De Boeck.

Saukko, P. (2003). Doing research in cultural studies. An introduction to classical and new methodological approaches. SAGE.

Spitzberg, B. H. et Changnon, G. (2009). Conceptualizing intercultural competence. Dans D. K. Deardorff (dir.), The SAGE handbook of intercultural competence (p. 2-52). SAGE.

Sue, D. W. (2010). Microaggressions in everyday life. Race, gender, sexual orientation. Wiley.

White, B. et Gratton, D. (2017). L'atelier de situations interculturelles : une méthodologie pour comprendre l'acte à poser en contexte pluriethnique. Alterstice, 7(1), 63-76.

Zembylas, M. (2010). Teachers' emotional experiences of growing diversity and multiculturalism in schools and the prospects of an "ethic of discomfort". Teachers and Teaching: theory and practice, 16(6), 703-716. https://doi.org/10.1080/13540602.2010.517687

Zembylas, M. et Papamickael, E. (2017). Pedagogies of discomfort and empathy in multicultural education. Intercultural Education, 28(1), 1-19. https://doi.org/10.1080/14675986.2017.1288448

\section{NOTES}

1. Le terme écrit en anglais est conservé tout au long de l'article. Il est central dans le projet photographique de Kim Kyun (2013) sur lequel nous nous appuyons. 
2. Nous remercions chaleureusement Kim Kyun, l'autrice et propriétaire des photographies du projet universitaire, de nous avoir accordé le droit d'utiliser ses œuvres dans le cadre de cet article scientifique pour la revue RIPES (échanges courriels du 10 juin 2020).

3. Dans son livre, Sue ajoute les Native Americans qui ne sont pas représentés dans la série de photos du projet Kim Kyun

4. Terme traduit de l'anglais (Goffman est un sociologue américain)

\section{ABSTRACTS}

In this article, after having exposed a training device, we show through the results of a study, how students teachers develop intercultural competences (IC) in negotiated collective and individual writings about experienced microaggressions (Kyun, 2013). The students, working part-time in a classroom, are confronted with heterogeneous contexts in schools and subject to injunctions to respect others. Many researchers define IC as a reflexive and (self-)critical process that considers the context in which interlocutors interact. In an MEEF master's degree programme at a university in the Hauts-de-France, the pedagogical system of intercultural training is designed in coherence with this paradigm so that students develop ICs. The innovative training device challenges the dominant ideology surrounding ICs, namely the militancy for an idealized living together, through dialogue between students. Finally, the points of vigilance presented open doors for other university courses.

Dans cet article, après avoir exposé un dispositif pédagogique universitaire, nous montrons à travers les résultats d'une étude, comment les étudiants futurs enseignants développent des compétences interculturelles ( $\mathrm{CI}$ ) dans des productions collectives négociées et dans des écrits individuels autour de microaggressions ${ }^{1}$ vécues (Kyun, 2013). Les étudiants, en poste à mi-temps dans une classe, sont confrontés à des contextes hétérogènes dans les écoles, et assujettis à des injonctions au respect d'autrui. De nombreux chercheurs définissent la notion de CI comme un processus réflexif et (auto)critique qui tient compte des contextes situés dans lesquels les interlocuteurs interagissent. Dans un master Métiers de l'Enseignement, de l'Éducation et de la Formation (MEEF) d'une université des Hauts-de-France, le dispositif pédagogique d'une formation interculturelle est conçu en cohérence avec ce paradigme pour que les étudiants y développent des CI. Innovant, le dispositif bouscule l'idéologie dominante qui entoure les CI, à savoir le militantisme pour un vivre ensemble idéalisé, par le dialogue entre les étudiants. Enfin, les points de vigilance présentés ouvrent des portes pour d'autres parcours universitaires.

INDEX

Mots-clés: enseignants stagiaires, inconfort, réflexivité, projet Kyun, microaggressions

\section{AUTHOR}

\section{VÉRONIQUE LEMOINE-BRESSON}

ATILF CNRS Université de Lorraine, Nancy, France, veronique.lemoine-bresson@univ-lorraine.fr 